\title{
COMPLEMENTO AD UNA NOTA DEL SIG. EMCH.
}

\author{
Nota di Giulio Giraud (Torino).
}

Adunanza del 23 giugno $190 \%$.

Il sig. Емсн, nella sua Nota: The Configurations of the Points of Inflexion of a Plane Cubic and their Harmonic Polars [questi Rendiconti, t. XXIII ( ${ }^{\circ}$ semestre 1907), pp. 25I-254], enuncia, come ultimo risultato, che esistono 8I rette ciascuna delle quali contiene un punto d'inflessione e due punti di contatto di tangenti condotte da flessi, e che per ogni punto d'inflessione passano 9 di tali rette. Questo risultato va corretto nel senso che il numero di tali rette è ro8 e che per ogni flesso passano I 2 rette siffatte.

Infatti, fissiamo un flesso $F$ e consideriamo la retta che lo unisce al punto $T$ di contatto d'una tangente $t$, uscente da un altro flesso $G$. Consideriamo l'omologia armonica di centro $F$ che muta in sè la cubica. Da essa il flesso $G$ è mutato in un altro flesso $G^{\prime}$ e le tangenti da $G$ alla cubica sono mutate in tangenti da $G^{\prime}$ alla cubica. Ciò accadrd in particolare per la $t$ che sard mutata in una tangente $t^{\prime}$; il punto $T \mathrm{di}$ contatto della $t$ sard mutato nel punto $T^{\prime}$ di contatto della $t^{\prime}$; onde la retta $F T$ dovrd passare per $T^{\prime}$. Ne viene che, congiungendo ogni flesso con i punti di contatto delle 24 tangenti alla cubica condotte dagli altri flessi e diverse dalle tangenti d'inflessione, le rette che si ottengono contengono sempre un altro di tali punti di contatto, onde tali rette uscenti da un flesso sono precisamente $\mathrm{I} 2$, e quindi il numero totale delle rette per un flesso contenenti due punti di contatto di tangenti condotte da altri flessi è ıo8.

Che d'altronde sia $\mathrm{I} 2$ e non 9 il numero di tali rette uscenti da un flesso, onde I08 e non 8I il numero totale di esse, risulta pure dalla tabella posta a pag. 252 della Nota del sig. Eмсн. Egli infatti alla fine della pagina 253 da il gruppo:

di terne di punti allineati.

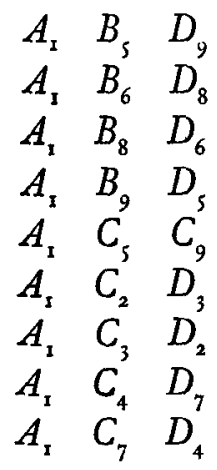


Ma dalla tabella risulta che oltre a quelle 9 terne di punti allineati contenenti $A_{\mathrm{s}}$ si debbono considerare le terne

$$
\begin{array}{lll}
A_{5} & B_{2} & B_{3} \\
A_{1} & B_{4} & B_{7} \\
A_{\mathrm{I}} & C_{6} & C_{8},
\end{array}
$$

onde 12 e non 9 sono le rette di cui ci occupiamo uscenti da $A_{\mathrm{r}}$ e quindi da ogni flesso.

Torino, 13 giugno 1907.

Givilo Giraud. 